\title{
Pesquisas Qualitativas usando Grounded Theory
}

\author{
Cleidson R. B. de Souza ${ }^{1}$, Igor Steinmacher ${ }^{2,3}$, Rafael Prikladnicki ${ }^{4}$ \\ 1 Universidade Federal do Pará - UFPA \\ ¿Universidade Tecnológica Federal do Paraná - UTFPR \\ ${ }^{3}$ Northern Arizona University - NAU \\ 4Pontifícia Universidade Católica do Rio Grande do Sul - PUCRS \\ cleidson.desouza@acm.org
}

\begin{abstract}
Este documento descreve a proposta de um minicurso sobre pesquisa qualitativas usando o método Grounded Theory.
\end{abstract}

\section{Keywords}

Grounded theory, teoria fundamentada em dados, métodos qualitativos.

\section{APRESENTAÇ̃̃̃O}

A Grounded Theory (GT), ou "Teoria Fundamentada em Dados" é uma metodologia de pesquisa que usa uma técnica indutiva baseada na análise sistemática e incremental de dados qualitativos (Strauss \& Corbin, 1998). Ela é uma metodologia amplamente utilizada em disciplinas das ciências sociais (Sociologia, Antropologia, etc) e até mesmo ciências médicas (Enfermagem e Medicina). Dado o aumento de estudos qualitativos em Ciência da Computação, a GT mostra-se cada vez mais importante por facilitar a transição de métodos quantitativos para métodos qualitativos. Ao mesmo tempo em que cresce o uso da GT, muitos pesquisadores acabam executando o método de maneiras distorcidas ou até mesmo equivocadas (Suddaby, 2006). Neste sentido, este minicurso tem por objetivo apresentar de forma teórica, porém com exemplos práticos, a GT como uma metodologia para condução de pesquisas qualitativas em ciência da computação. $\mathrm{O}$ minicurso abordará o histórico do método, seus conceitos e exemplos práticos de uso. Ao final do minicurso os participantes deverão ser capazes de: entender e analisar a viabilidade e a adequação do método de Grounded Theory e aplicar o método de maneira apropriada.

\section{SUMÁRIO ESTENDIDO}

O minicurso será dividido em quatro partes. A primeira parte visa contextualizar o método de GT dentre outros métodos de pesquisa qualitativa e quantitativa. Ele prevê a comparação do

Permission to make digital or hard copies of all or part of this work for personal or classroom use is granted without fee provided that copies are not made or distributed for profit or commercial advantage and that copies bear this notice and the full citation on the first page. Copyrights for components of this work owned by others than the author(s) must be honored. Abstracting with credit is permitted. To copy otherwise, or republish, to post on servers or to redistribute to lists, requires prior specific permission and/or a fee. Copyright 2018 SBC.

IHC 2018, Anais Estendidos do XVII Simpósio Brasileiro sobre Fatores Humanos em Sistemas Computacionais.

Outubro 22-26, 2018, Belém, Brasil

Minicursos método com outras abordagens tradicionais da literatura, como experimentos, surveys, etc. Desta forma, esta parte inicial visa fornecer à audiência um entendimento mais completo sobre quando utilizar a GT. A segunda parte apresenta uma introdução à GT, explicando os seus conceitos básicos e, principalmente, enfatizando a parte mais importante do método: a codificação de dados. A terceira parte do minicurso explica as 3 fases da GT, a saber: codificação aberta, axial e seletiva. Nesta parte, diversos exemplos serão utilizados para ilustrar a adequada aplicação da GT. Finalmente, a quarta e última parte do minicurso visa apresentar as conclusões do minicurso, finalizando com uma discussão sobre o potencial do método em pesquisas qualitativas e outros temas em aberto. Pretende-se também mencionar brevemente ferramentas automatizadas de apoio a utilização da GT.

Parte I - Introdução aos Métodos Qualitativos (30 minutos): Nesta fase inicial serão feitas considerações gerais sobre metodologias de pesquisa em Ciência da Computação. Por exemplo, serão apresentadas as principais diferenças entre os diferentes tipos de métodos: experimentais $\mathrm{x}$ analíticos; quantitativos $\mathrm{x}$ qualitativos e primários $\mathrm{x}$ secundários. Exemplos de contextos de utilização destes diferentes tipos de métodos serão apresentados, com ênfase na motivação para uso dos métodos qualitativos. Finalmente, será apresentado o contexto de uso do método de Grounded Theory (GT).

Parte II - Introdução a GT (30 minutos): O Histórico da GT. Principais conceitos: codificação, comparação constante e saturação teórica. Exemplos de utilização da GT em pesquisas em diversas áreas e exemplos de resultados obtidos usando a GT em Interação Humano-Computador, Engenharia de Software e Sistemas Colaborativos. Um exemplo de planejamento de estudo da GT e as principais fases da pesquisa ao utilizar a mesma serão apresentados. Por exemplo, pretende-se mencionar a necessidade de se intercalar períodos de coleta e análise de dados visando obter saturação teórica dos dados.

Parte III - GT em detalhes (105 minutos): Abordagens para coleta de dados no contexto da GT. Análise de dados qualitativos usando a GT, detalhando as três fases da codificação da GT: codificação aberta (atribuição de conceitos para os fenômenos de interesse e criação de categorias que agregam estes conceitos); codificação axial (identificação de relacionamentos entre esses conceitos); e codificação seletiva (identificação da categoria central da teoria, com a qual todas as outras estão relacionadas). Descrição da abordagem, apresentação de exemplos teóricos e práticos para cada uma das três fases da codificação. 
Parte IV - Considerações Finais (15 minutos): Breve apresentação de ferramentas de apoio a análise qualitativa de dados (MaxQDA2 e Atlas.TI) e de outros exemplos de uso de GT em pesquisas de Interação Humano-Computador, Engenharia de Software e Sistemas Colaborativos. Discussão sobre as limitações da GT. Discussão sobre as principais referências bibliográficas incluindo referencias com exemplos de utilização da GT.

\section{PÚBLICO-ALVO e TIPO}

Este minicurso é dirigido a pesquisadores (professores, alunos de pós-graduação iniciantes ou avançados, alunos de graduação avançados e pesquisadores na indústria) que desejam entender sobre o efetivo uso de Grounded Theory como um método qualitativo de pesquisa em Ciência da Computação.

\section{BIOGRAFIA DOS AUTORES}

Os três autores deste minicurso utilizaram o método de GT durante seus cursos de doutorados (entre 2000 e 2014), com resultados da utilização deste método publicados em periódicos, conferências e workshops de prestígio. Mais detalhes abaixo.

Cleidson R. B. de Souza. É professor da Faculdade de Computação da Universidade Federal do Pará. Ele obteve seu mestrado na Unicamp em 1998. Em 2005 o grau de Doutor pelo Department of Informatics da University of California, Irvine. Ele foi pesquisador visitante da N.A.S.A. Ames Research Center (2002), IBM T. J. Watson Research Center (2003 e 2004), University of California, Irvine (2006 e 2007). Ele também foi pesquisador da IBM Research Brazil (2010-2011) do Instituto Tecnológico Vale (2012-2018). Ele é membro da Sociedade Brasileira de Computação (SBC) e da Association for Computer Machinery (ACM). Ele foi eleito membro afiliado da Academia Brasileira de Ciências para o período de 2014-2018. Eles possui diversos artigos publicados nos melhores periódicos e eventos nacionais e internacionais onde utiliza pesquisa qualitativa.

Lattes: http://lattes.cnpq.br/6490014244112888

Igor Steinmacher. É professor adjunto do Departamento de Computação da Universidade Tecnológica Federal do Paraná, atualmente em período de estágio pós-doutorado na Northern Arizona University. Ele obteve seu mestrado em Ciência da Computação na UFRGS em 2005, e seu título de Doutor em Ciência da Computação pelo Instituto de Matemática e Estatística da Universidade de São Paulo, em 2015. Ele participa ativamente de conferências da área de Sistemas Colaborativos e Engenharia de Software e de Comissões Especiais da SBC. Possui diversos artigos publicados em periódicos e eventos nacionais e internacionais relacionados a aspectos sociais e humanos da engenharia de software, sistemas colaborativos, mineração de repositórios de software e educação em engenharia de software.

Currículo Lattes: http://lattes.cnpq.br/5529725593221391

Rafael Prikladnicki. Professor do Programa de Pós-Graduação em Ciência da Computação da Faculdade de Informática da PUCRS e coordenador adjunto do grupo de pesquisa em Desenvolvimento Distribuído de Software (MuNDDoS) na mesma Universidade. Mestre e Doutor em Ciência da Computação pela PUCRS, com estágio de doutorado no Departamento de Ciência da Computação da Universidade de
Victoria, em British Columbia, Canadá. Seus interesses em pesquisa incluem desenvolvimento distribuído de software, gerência de projetos de software com ênfase em gerência de risco, qualidade de software, metodologias ágeis para desenvolvimento de software, e engenharia de software experimental. Tem participado ativamente de grupos de interesse sobre pesquisas qualitativas em Engenharia de Software, além de ter publicado artigos sobre o tema em eventos nacionais e internacionais. Membro da Sociedade Brasileira de Computação (SBC) e entidades internacionais da área (AIS, ACM, IEEE e PMI). Possui diversos artigos publicados em periódicos e eventos nacionais e internacionais relacionados com as suas áreas de interesse.

Currículo Lattes: http://lattes.cnpq.br/2007065934836962

\section{DURAÇÃO E IDIOMA}

O minicurso proposto deve ter duração de 3 horas e será apresentado em Português.

\section{INFRAESTRUTURA NECESSÁRIA}

Datashow, micro-computador equipado com Microsoft PowerPoint e caixa de som.

\section{CONFERÊNCIAS OU SEMINÁRIOS ONDE O MINICURSO JÁ FOI OU SERÁ APRESENTADO.}

Um minicurso específico sobre Pesquisa Qualitativa com Grounded Theory foi apresentado em Junho de 2010 pelos Profs. Cleidson e Rafael no Simpósio Brasileiro de Qualidade de Software, em Belém. Este minicurso contou com a participação de 72 pessoas entre alunos e professores, sendo um dos tutoriais com maior público. Este mesmo minicurso foi apresentado em Outubro de 2010 no Congresso Brasileiro de Software, em Salvador, com a participação de mais de 35 pessoas, tendo novamente sido um dos tutoriais com maior participação. Mais recentemente, em 2015, o prof. Igor Steinmacher apresentou um minicurso sobre o tema na Escola Avançada de Qualidade de Software na Amazônia, em Manaus para um público aproximado de 100 pessoas. Finalmente, este minicurso foi apresentado durante o IHC 2017 em Joinville. Pretende-se repetir o mesmo este ano pois o primeiro autor está localizado em Belém e não precisará se deslocar para o mesmo.

\section{REFERENCES}

[1] BANDEIRA-DE-MELLO, R., CUNHA, C., 2006. "Grounded Theory". In: Godoi, C. K., Bandeira-de-Mello, R., Silva, A. B. d. (eds), Pesquisa Qualitativa em Estudos Organizacionais: Paradigmas, Estratégias e Métodos, Chapter 8, São Paulo, Saraiva.

[2] GLASER, B., 1992. "Basics of grounded theory analysis". Mill Valley CA, The Sociology Press.

[3] GLASER, B., STRAUSS, A., 1967. "The discovery of grounded theory: Strategies for Qualitative Research". New York, Aldine Transaction.

[4] OATES, B. J., 2006. "Researching Information Systems and Computing”, London: Sage Publications. 
[5] STRAUSS, A., 1987. "Qualitative analysis for social scientists". New York, Cambridge University Press.

[6] STRAUSS, A., CORBIN, J., 1998. "Basics of Qualitative Research: Techniques and Procedures for Developing Grounded Theory". 2 ed. London, SAGE Publications.
[7] SUDDABY, R., 2006. "From the Editors: What Grounded Theory is Not," Academy of Management Journal, vol. 49, no. 4, pp. 633-642 